\title{
EXTENSÃO DO MODELO F-SAC PARA GASES LEVES VIA UMA REGRA DE MISTURA E EQUAÇAO CÚBICA DE ESTADO
}

\author{
G. B. FLÔRES, P. B. STAUDT e R. de P. SOARES \\ Universidade Federal do Rio Grande do Sul, Departamento de Engenharia Química \\ E-mail para contato: \{gbflores, rafael\}@enq.ufrgs.br
}

\begin{abstract}
RESUMO - O modelo preditivo de coeficiente de atividade F-SAC, recentemente proposto, une características do conceito de grupos funcionais com a teoria COSMO-RS. A principal vantagem deste modelo é o reduzido número de parâmetros de interação quando comparado ao modelo UNIFAC e variantes. Para a utilização do F-SAC em sistemas contendo gases leves uma alternativa é a sua associação com uma equação de estado cúbica através de uma regra de mistura. No presente trabalho a regra de mistura SCMR foi utilizada em conjunto com o modelo F-SAC e a equação SRK para o cálculo de equilíbrio de fases de sistemas envolvendo gases leves. Novos grupos funcionais foram adicionados ao modelo e seus parâmetros estimados. Bons resultados com o modelo combinado foram encontrados em comparação com dados experimentais em amplas faixas de pressão e temperatura. Os resultados do modelo proposto também foram comparados com outros modelos disponíveis na literatura.
\end{abstract}

\section{INTRODUÇÃO}

Atualmente, pelo menos para fins de engenharia, os modelos preditivos de maior sucesso para o cálculo de coeficientes de atividade são os baseados em grupos funcionais, tais como UNIFAC (UNIversal quasichemical Functional group Activity Coefficients model) e suas variantes (GRENSEMANN e GMEHLING, 2005). Entretanto, por utilizarem parâmetros binários entre os grupos funcionais, estes modelos requerem uma grande quantidade de dados experimentais para o ajuste das suas matrizes de parâmetros, fazendo com que estas cresçam com o quadrado do número de grupos. Já os modelos do tipo COSMO-RS (COnductor-like Screening MOdel for Real Solvents) (KLAMT et. al. 2010) requerem a calibração de um pequeno grupo de parâmetros universais, porém ainda não contam com a precisão necessária para cálculos de otimização de sistemas de separação, por exemplo.

Explorando estas oportunidades, recentemente um novo modelo foi proposto pelo presente grupo de pesquisa, chamado de F-SAC (Functional-Segment Activity Coefficient) (SOARES e GERBER, 2013). Este modelo também é baseado no conceito de grupos funcionais, mas a energia de interação entre os grupos vem da teoria COSMO-RS (KLAMT, 1995). A princípio, uma vez ajustados os parâmetros de cada grupo, estes funcionariam para descrever a interação para qualquer par de grupos. Esta é a principal vantagem do modelo F-SAC, o número de parâmetros do modelo cresce proporcionalmente ao número de grupos (pelo menos quando não há formação de ligação de 
hidrogênio).

Porém, como o modelo F-SAC é na verdade um modelo de energia livre de Gibbs em excesso, originalmente desenvolvido para líquidos, este não pode representar adequadamente gases leves e fluidos supercríticos. Sendo assim, o presente trabalho tratou da combinação do modelo F-SAC com a equação cúbica de estado SRK. Para tal, foi utilizada a regra de mistura também desenvolvida previamente pelo presente grupo de pesquisa, conhecida como SCMR (Self-Consistent Mixing Rule) (STAUDT e SOARES, 2012).

\section{MODELOS UTILIZADOS E METODOLOGIA}

\subsection{O Modelo F-SAC}

No modelo F-SAC, bem como em todas as variantes dos modelos UNIFAC e COSMO-RS, o coeficiente de atividade da fase líquida é calculado como função da temperatura e composição pela soma de componentes residual e combinatorial. A contribuição combinatorial, que considera diferenças de forma e tamanho da molécula dos componentes, é similar a que está presente no UNIFAC (Do), e foi originalmente desenvolvida por Soares, (2011) para ser utilizada em modelos COSMO-SAC (LIN e SANDLER, 2002). A contribuição residual, que representa as interações moleculares observadas na mistura, é idêntica à que está presente no modelo COSMO-SAC, uma variação do modelo COSMO-RS. Nestes modelos é utilizada uma teoria de superfícies de contato, onde cada trecho ou contato da superfície da molécula apresenta uma carga aparente e a energia entre dois contatos é calculada com base nestas cargas, da seguinte forma:

$$
\Delta W\left(\sigma_{m}, \sigma_{n}\right)=\theta(T, m, n) \frac{\alpha^{\prime}\left(\sigma_{m}+\sigma_{n}\right)^{2}}{2}+\frac{E^{H B}\left(\sigma_{m}, \sigma_{n}\right)}{2}
$$

onde a primeira contribuição é calculada em termos dos contatos $m$ e $n$ e a segunda contribuição $E^{H B}$ é a energia da ligação de hidrogênio entre os segmentos.

A grande vantagem do F-SAC é que o primeiro termo da Equação 1 depende apenas de parâmetros dos grupos funcionais e apenas o $E^{H B}$ depende do par de grupos envolvidos. Como o número de grupos funcionais é bem maior que o número de grupos que podem formar ligações de hidrogênio tem-se um número reduzido de parâmetros. Mais detalhes podem ser encontrados em trabalhos anteriores (POSSANI et al., 2014; e SOARES e GERBER, 2013).

\subsection{Regra de Mistura (SCMR)}

A combinação de equações de estado (EoS - Equations of State) cúbicas com modelos de energia livre de Gibbs em excesso ( $G^{E}$ ) é bem conhecida. Desde a ideia básica de Huron e Vidal, (1979) várias regras de misturas foram desenvolvidas para estender a aplicação de EoS cúbicas. Quando EoS cúbicas são combinadas com modelos de $G^{E}$ preditivos (por exemplo, UNIFAC e variantes, modelos do tipo COSMO-RS e F-SAC) os modelos resultantes podem se tornar poderosas 
ferramentas preditivas para uma grande gama de aplicações.

A regra de mistura SCMR (Self-Consistent Mixing Rule) foi desenvolvida pelo presente grupo de pesquisa (STAUDT e SOARES, 2012) e testada no trabalho original com uma grande variedade de modelos de coeficiente de atividade (Wilson, UNIQUAC, UNIFAC (Do) e COSMO-SAC). Para esta regra de mistura, a combinação da equação de estado e modelo de $G^{E}$ se dá em termos do parâmetro $q=a / b R T$ da equação cúbica, da seguinte forma:

$$
q=\frac{1}{A_{0}}\left(\frac{G_{\gamma}^{E}}{R T}+\sum_{i} x_{i} \ln \frac{b}{b_{i}}\right)+\sum_{i} x_{i} q_{i}
$$

onde $A_{0}$ é uma constante, característica da equação cúbica de estado escolhida. Mais detalhes podem ser encontrados no trabalho original (STAUDT e SOARES, 2012).

\subsection{Estimação de parâmetros}

A principal vantagem da SCMR com relação às demais regras de mistura é que esta reproduz muito bem o modelo de atividade original em baixas pressões. Assim, no presente trabalho todos os parâmetros originais do modelo F-SAC, conforme apresentados por (POSSANI et al., 2014), puderam ser utilizados sem alterações. Isto não seria possível com outras regras de mistura, como é o caso da regra de mistura PSRK, onde o modelo UNIFAC (Do) é utilizado, porém com tabelas de parâmetros diferentes. Novos grupos funcionais foram introduzidos no presente trabalho seguindo o procedimento de seleção de grupos recomendado em (GERBER, 2012). Neste trabalho foram introduzidos 2 novos grupos funcionais para as moléculas de $\mathrm{CO}_{2}$ e $\mathrm{NH}_{3}$ e um novo subgrupo para o $\mathrm{CH}_{4}$, derivado do grupo de hidrocarbonetos lineares. Ao total, neste trabalho foram calibrados 17 novos parâmetros.

A calibração seguiu a função objetivo de desvio absoluto médio relativo (AARD - absolute average relative deviation) para dados de VLE, da seguinte forma:

$$
\operatorname{AARD}_{V L E}=\frac{1}{\mathrm{NP}} \sum_{i}^{\mathrm{NP}} \frac{\left|P_{i}^{\text {exp }}-P_{i}^{\text {calc }}\right|}{P_{i}^{\exp }}
$$

onde NP é o numero de pontos experimentais, $P_{i}^{\exp }$ e $P_{i}^{\text {calc }}$ são respectivamente a pressão experimental e a calculada pelo modelo.

Para os valores calculados foi utilizada uma rotina de ponto de bolha implementada previamente pelo grupo e que opera com diversas equações cúbicas e regras de mistura, incluindo a SCMR. Foi necessário apenas combinar a SCMR com o modelo F-SAC. O método de otimização utilizado foi do tipo poliedros flexíveis, similar ao descrito nos trabalhos anteriores do modelo F-SAC (GERBER, 2012; POSSANI et al., 2013). 


\section{RESULTADOS E DISCUSSÃO}

\subsection{Parâmetros F-SAC para gases leves}

Na Tabela 1 estão apresentados os parâmetros do modelo F-SAC estimados neste trabalho para os grupos funcionais e subgrupos adicionados. Na Tabela 2 são mostrados os parâmetros referentes a ligações de hidrogênio para a amônia e alguns dos outros grupos já existentes no modelo.

Tabela 1: Novos parâmetros do modelo F-SAC estimados neste trabalho.

\begin{tabular}{|l|l|l|l|l|l|l|l|}
\hline Grupo & $Q_{k}^{+} / \AA^{2}$ & $Q_{k}^{-} / \AA^{2}$ & $\sigma_{k}^{+} / e \mathrm{em}^{-2}$ & $\beta_{k} \times 10^{6} K$ & Subgrupo & $R_{k} / \AA^{3}$ & $Q_{k} / \AA^{2}$ \\
\hline CH2 & 0 & 0 & 0 & 209.26 & $\mathrm{CH} 4$ & 40.47 & 109.22 \\
\hline NH3 & 9.438 & 12.18 & $1.032 \cdot 10^{-2} 2$ & 200.00 & NH3 & 50.86 & 122.786 \\
\hline CO2 & 47.637 & 10.874 & $2.425 \cdot 10^{-3}$ & 200.00 & CO2 & 33.84 & 56.825 \\
\hline
\end{tabular}

Tabela 2: Parâmetros de ligação de hidrogênio para NH3 e alguns outros grupos.

\begin{tabular}{|l|l|l|l|}
\hline Doador & Receptor & \multicolumn{1}{|c|}{$\Delta W^{H B}\left(\sigma_{m}, \sigma_{n}\right) / \mathrm{kJ}^{\prime} \cdot \mathrm{mol}^{-1}$} & \multicolumn{1}{|c|}{$\psi_{H B}$} \\
\hline $\mathrm{NH} 3$ & $\mathrm{NH} 3$ & 4.929 & 0.215 \\
\hline $\mathrm{NH} 3$ & $\mathrm{H} 2 \mathrm{O}$ & 7.266 & 0.66 \\
\hline $\mathrm{H} 2 \mathrm{O}$ & $\mathrm{NH} 3$ & 5.222 & -0.076 \\
\hline ACH & NH3 & 0.665 & -0.226 \\
\hline
\end{tabular}

\subsection{Resultados dos VLE}

Para ilustrar a importância do uso de uma equação de estado em conjunto com uma regra de mistura baseada em modelos de $G^{E}$, são apresentados na Figura 1 os diagramas de fase de misturas de (a) etano - propileno e (b) metano - propano. O equilíbrio líquido-vapor foi calculado com o modelo F-SAC puro (em que a fase vapor é considerada como ideal utilizando a lei de Raoult modificada) e com a combinação do F-SAC com a equação cúbica de estado de Soave-RedlichKwong com a extensão de Mathias-Copeman (MATHIAS e COPEMAN, 1983) através da regra de mistura SCMR, chamado aqui de SRK(MC)-SCMR(F-SAC). Vale ressaltar que os parâmetros do modelo F-SAC foram os mesmos para as duas representações. Como pode ser visto na Figura 1(a), em baixas condições de temperatura e pressão, ambos os modelos apresentam resultados semelhantes e coerentes com os dados experimentais. Porém, na medida em que a pressão e temperatura do sistema aumentam, a fase vapor passa a não se comportar mais como um gás ideal e as correlações de pressão de saturação param de fazer sentido. $O$ resultado é que a representação pela lei de Raoult modificada passa a diferir bastante dos dados experimentais. 


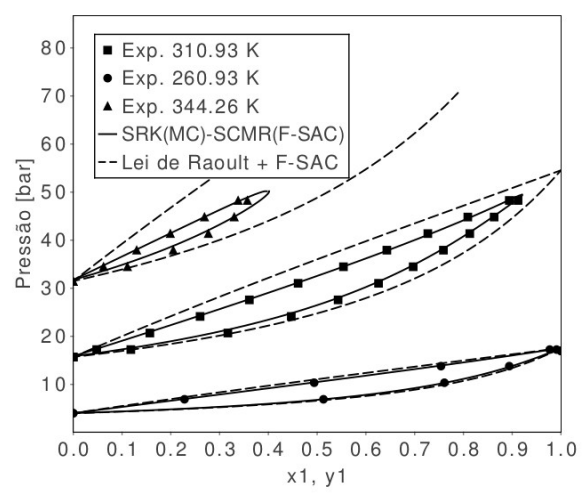

(a) etano - propileno

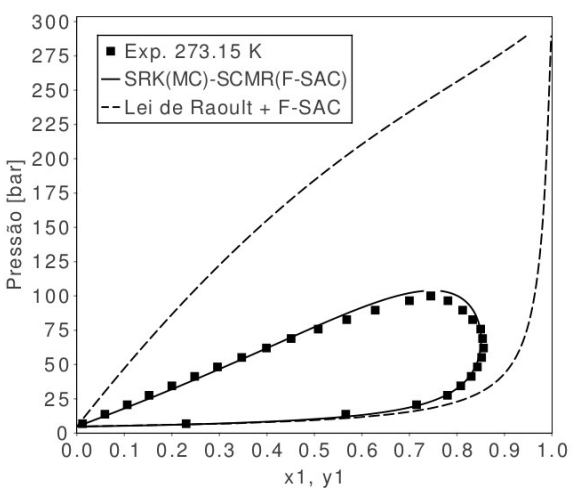

(b) metano - propano

Figura 1 - Diagrama de equilíbrio líquido-vapor para os sistemas (a) etano - propileno e (b) metano propano em alta pressão.

Na Figura 2 é mostrado o diagrama de fases de misturas contendo amônia, com benzeno e água respectivamente, calculados com os modelos SRK(MC)-SCMR(F-SAC), PSRK (CHEN et.al, 2002) e SRK(MC). Pode-se perceber que a EoS sozinha (com a regra de mistura clássica) não é capaz de representar com precisão adequada o VLE da mistura, possivelmente devido à grande diferença de comportamento entre as substâncias envolvidas: a amônia é uma molécula muito polar, inclusive com formação de ligação de hidrogênio.

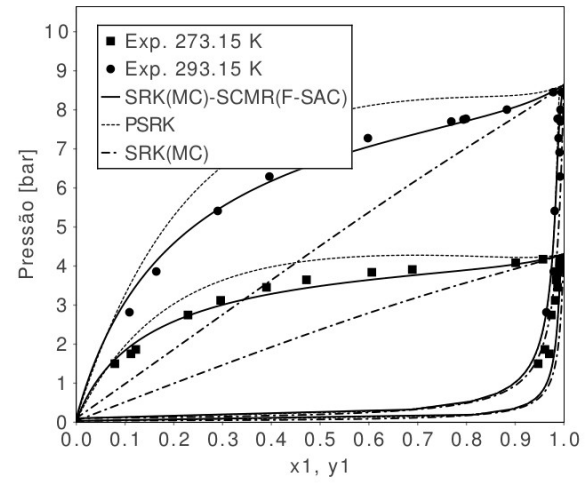

(a) amônia - benzeno

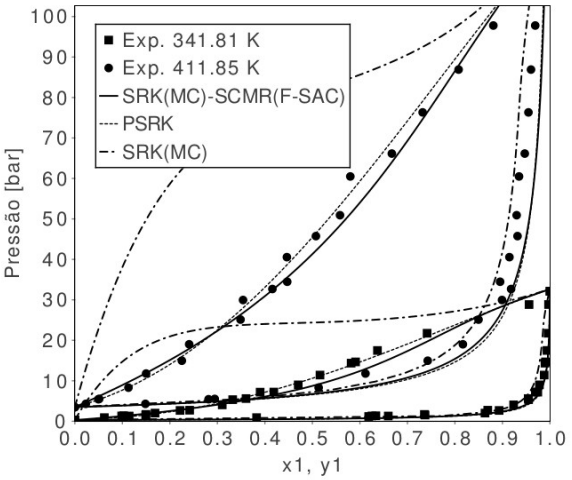

(b) amônia - água

Figura 2 - Diagrama de VLE para os sistemas (a) amônia - benzeno e (b) amônia - água em diferentes escalas de pressões.

Combinando a análise das Figuras 1 e 2, é possível imaginar que para a mistura amôniabenzeno, se o experimento ocorrer em uma temperatura e pressão mais altas, o modelo F-SAC puro (que trata a fase vapor como um gás ideal) não poderá representar a mistura com a mesma precisão, necessitando da combinação com a EoS através de uma regra de mistura. Ao mesmo tempo, a EoS sozinha não retém informações suficientes para representar adequadamente a mistura devido à grande diferença de comportamento das substâncias envolvidas mesmo em baixas pressões. Desta forma, pode-se perceber a vantagem do uso de EoS combinadas com modelos de atividade para misturas 
assimétricas e polares e condições de temperatura e pressão elevadas.

A Figura 3 mostra o diagrama de fases de misturas contendo $\mathrm{CO}_{2}$ e hidrocarbonetos em diferentes condições de temperatura e pressão com os mesmos modelos apresentados na Figura 2. Pode-se perceber novamente que a equação de estado sozinha não apresenta bons resultados enquanto as regras de mistura são mais coerentes com os dados experimentais.

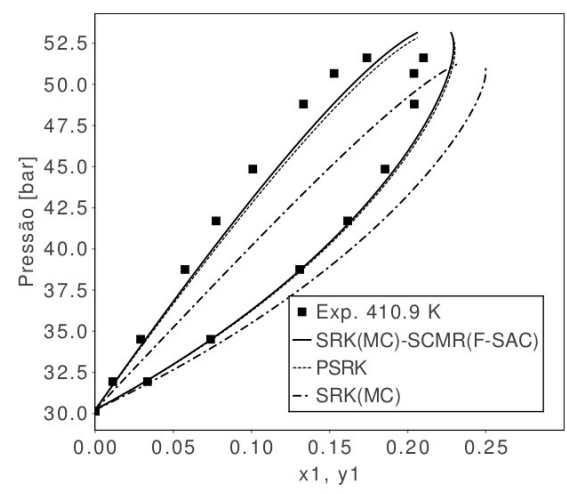

(a) $\mathrm{CO}_{2}-$ n-butano

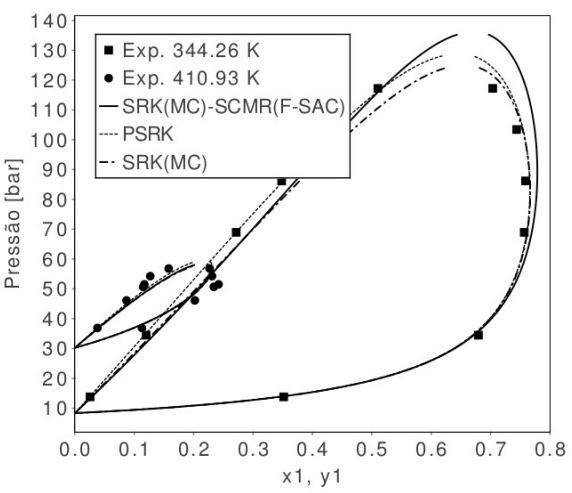

(b) $\mathrm{CO}_{2}-$ n-pentano

Figura 3 - Diagrama de fases de misturas contendo dióxido de carbono e hidrocarbonetos.

Na Figura 4 estão representados mais resultados para misturas de hidrocarbonetos. Devido à similaridade das substâncias envolvidas, tanto de polaridade quanto de tamanho, todos os modelos comparados foram capazes de representar as misturas. O conjunto SRK(MC)-SCMR(F-SAC) se mostrou preciso e sutilmente superior aos demais.

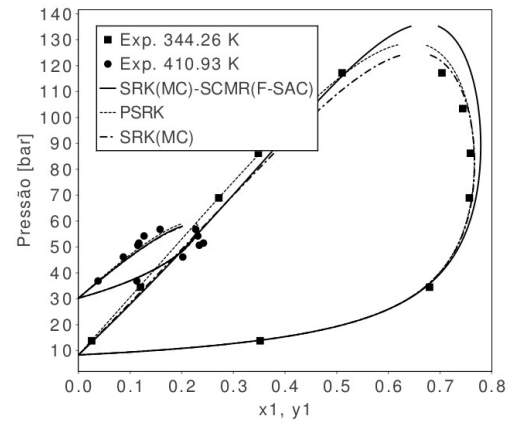

(a) metano - n-butano

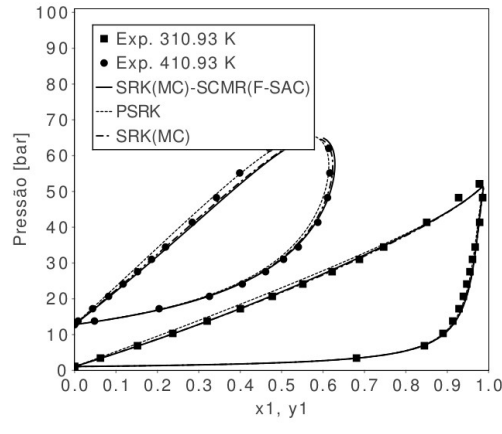

(b) etano-n-pentano

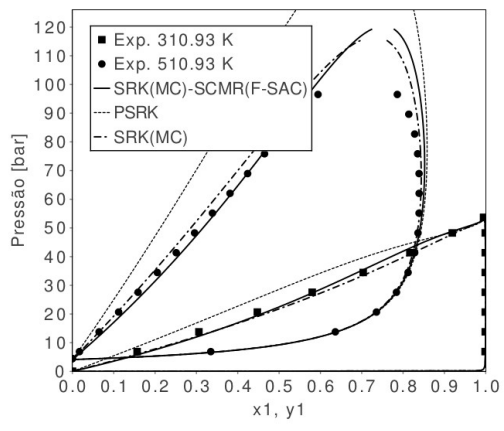

(c)etano - n-decano

Figura 4 - VLE das misturas (a) metano - n-butano, (b) etano-n-pentano e (c)etano - n-decano. 


\section{9 a 22 de outubro de 2014 \\ Florianópolis/SC}

\section{CONCLUSÃO}

Neste trabalho, o modelo F-SAC teve sua tabela de parâmetros estendida para representar equilíbrio líquido-vapor de gases leves em altas pressões e/ou temperaturas com o auxílio da equação de estado SRK com parâmetros de Mathias-Copeman (MC) através da regra de mistura SCMR (Self Consistent Mixing Rule). Os parâmetros originais do F-SAC foram mantidos constantes e apenas foram calibrados os parâmetros inseridos para a representação das novas substâncias $\left(\mathrm{CH}_{4}, \mathrm{NH}_{3} \mathrm{e}\right.$ $\mathrm{CO}_{2}$ ). Após a inserção dos novos grupos, resultados de predição de VLE de misturas contendo gases dissolvidos foram comparados com dados experimentais e com modelos da literatura (SRK(MC) e PSRK). Excelentes resultados foram obtidos até o momento mostrando o grande potencial do modelo combinado SRK(MC)-SCMR(F-SAC).

\section{BIBLIOGRAFIA}

CHEN, J.; FISCHER, K.; GMEHLING, J. Modification of PSRK mixing rules and results for vaporliquid equilibria, enthalpy of mixing and activity coefficients at infinite dilution. Fluid Phase Equilibria, v. 200, n. 2, p. 411-429, ago. 2002.

GERBER, R. P. NOVO MODELO DE COEFICIENTE DE ATIVIDADE: F-SAC. [s.1.] UFRGS, 2012.

GRENSEMANN, H.; GMEHLING, J. Performance of a Conductor-Like Screening Model for Real Solvents Model in Comparison to Classical Group Contribution Methods. Industrial \& Engineering Chemistry Research, v. 44, n. 5, p. 1610-1624, mar. 2005.

HURON, M.-J.; VIDAL, J. New mixing rules in simple equations of state for representing vapourliquid equilibria of strongly non-ideal mixtures. Fluid Phase Equilibria, v. 3, n. 4, p. 255-271, 1979.

KLAMT, A. Conductor-like Screening Model for Real Solvents: A New Approach to the Quantitative Calculation of Solvation Phenomena. The Journal of Physical Chemistry, v. 99, n. 7, p. 2224-2235, fev. 1995.

KLAMT, A.; ECKERT, F.; ARLT, W. COSMO-RS: an alternative to simulation for calculating thermodynamic properties of liquid mixtures. Annual review of chemical and biomolecular engineering, v. 1, p. 101-22, jan. 2010.

LIN, S.-T.; SANDLER, S. I. A Priori Phase Equilibrium Prediction from a Segment Contribution Solvation Model. Industrial \& Engineering Chemistry Research, v. 41, n. 5, p. 899-913, mar. 2002.

POSSANI, L. F. K. et al. Simultaneous Correlation of Infinite Dilution Activity Coefficient,VaporLiquid, and Liquid-Liquid equilibrium data with F-SAC. Fluid Phase Equilibria, v. 364, p. 31-41, dez. 2014. 
SOARES, R. DE P. The Combinatorial Term for COSMO-Based Activity Coefficient Models. Industrial \& Engineering Chemistry Research, v. 50, n. 5, p. 3060-3063, 2 mar. 2011.

SOARES, R. DE P.; GERBER, R. P. Functional-Segment Activity Coefficient Model. 1. Model Formulation. Industrial \& Engineering Chemistry Research, v. 52, n. 32, p. 11159-11171, 14 ago. 2013.

STAUDT, P. B.; SOARES, R. D. P. A self-consistent Gibbs excess mixing rule for cubic equations of state. Fluid Phase Equilibria, v. 334, p. 76-88, nov. 2012.

STAUDT, P. B.; SOARES, R. D. P. A self-consistent Gibbs excess mixing rule for cubic equations of state. Fluid Phase Equilibria, v. 334, p. 76-88, nov. 2012. 